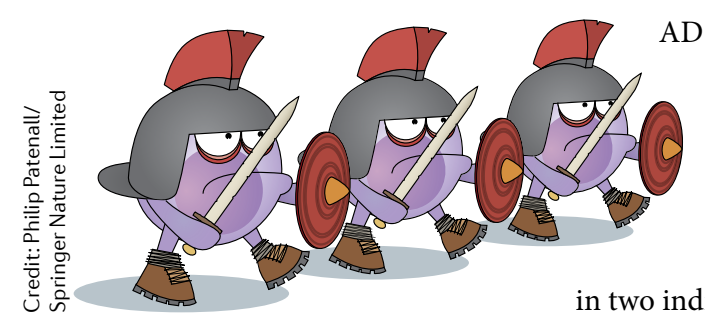

$\mathrm{AD}$ or MCI, whereas clonal expansion occurred less frequently in healthy individuals.

TCRs specific for Epstein-Barr virus (EBV) were observed in two individuals with $\mathrm{AD}$ and one individual with MCI. However, the researchers emphasize that

with immunological memory and secrete pro-inflammatory and cytotoxic molecules. $\mathrm{CD}^{+} \mathrm{T}$ cells that could have been $\mathrm{T}_{\mathrm{EMRA}}$ cells were also detected close to amyloid- $\beta$ plaques in post-mortem brain samples from individuals with $\mathrm{AD}$.

The researchers then performed single-cell RNA sequencing of immune cells in the CSF. "To our knowledge, we are the first to sequence CSF cells from individuals with $\mathrm{AD}$," notes Gate. "The real technological advance of our study is that we were able to sequence TCRs within the CSF, which allowed us to evaluate clonal expansion of these cells."

The researchers identified numerous highly expanded $\mathrm{T}$ cell clones - including $\mathrm{T}_{\mathrm{EMRA}}$ cells patrolling the CSF of individuals with this finding does not provide evidence of a causal link between $\mathrm{EBV}$ infection and $\mathrm{AD}$.

"While we find that many TCRs in the CSF of individuals with $\mathrm{AD}$ have specificity against EBV, we also uncovered many expanded $\mathrm{T}$ cell clones with unknown specificity," explains Gate. "Our next goal is to uncover the antigen specificities of these highly expanded clones. This approach could uncover a novel biomarker or therapeutic target for AD."

ORIGINAL ARTICLE Gate, D. et al. Clonally expanded CD8 T cells patrol the cerebrospinal fluid in Alzheimer's disease. Nature 577, 399-404 (2020)

\section{Sarah Lemprière}

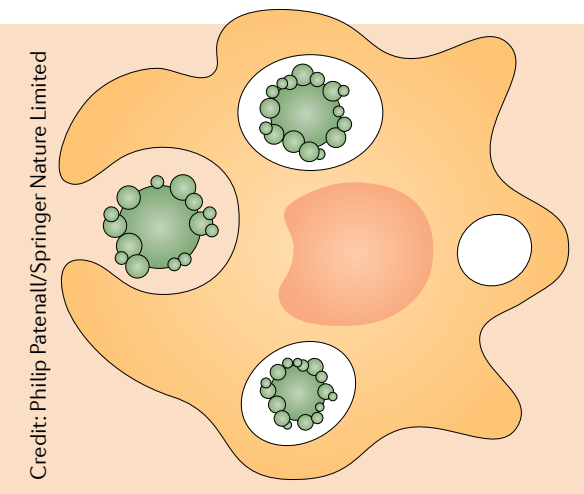

To investigate the role of RXR $\alpha$ in brain clean-up and stroke recovery, the researchers used in vitro $M \Phi$ cultures and a mouse model of ischaemic stroke. The in vitro experiments revealed that loss of RXRa impaired the ability of MФ to phagocytose dead neurons. In addition, mice with selective deletion of the RXRa gene in M $\Phi-$

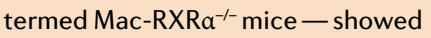
greater progression of brain atrophy and poorer functional recovery after stroke than their wild-type counterparts, despite similar initial infarct sizes.

The research team went on to examine the effects of augmentation of RXRa function of $M \Phi$, and as such RXR activation ... after stroke could enhance the clean-up and reduce the injury to the brain tissue caused by toxic by-products of ischaemic injury," write Aronowski and colleagues in their paper. "In conclusion, we propose that RXR could represent an attractive and clinically relevant target for improving post-stroke recovery through modification of $\mathrm{M} \Phi$."

\section{Heather Wood}

ORIGINAL ARTICLE Ting, S.-M. et al. Brain cleanup as a potential target for poststroke recovery: the role of RXR (retinoic $X$ receptor) in phagocytes. Stroke https://doi.org/10.1161/ STROKEAHA.119.027315 (2019) RELATED ARTICLE Hu, X. et al. Microglial and macrophage polarization - new prospects for brain repair. Nat. Rev. Neurol. 11, 56-64 (2015)

\section{IN BRIEF}

\section{$\rightarrow$ TRAUMATIC BRAIN INJURY \\ Nanoparticles improve outcomes of traumatic brain injury in mice}

Immunomodulatory nanoparticles (IMPs) could provide an acute treatment for traumatic brain injury (TBI) that would help to preserve function and improve recovery, according to a recent mouse study published in Annals of Neurology. The biodegradable biopolymer nanoparticles, which are approved by the FDA, bind to monocytes and prevent them from migrating to sites of inflammation. In TBI, monocyte-derived macrophages infiltrate the brain after injury and are involved in mediating secondary damage - blocking this process with IMPs could, therefore, protect against this process. Sripadh Sharma and colleagues tested this theory by administering IMPs to two different mouse models of TBI. Treatment with IMPs reduced immune infiltration into the brain and led to preservation of tissue and neurological function. The findings provide a rationale for translating this approach into the clinic as an acute treatment for TBI.

ORIGINAL ARTICLE Sharma, S. et al. Intravenous immunomodulatory nanoparticle treatment for traumatic brain injury. Ann. Neurol. https://doi.org/10.1002/ana.25675 (2020)

\section{$\Rightarrow$ ALZHEIMER DISEASE}

\section{Imaging shows predictive power of tau pathology in Alzheimer disease}

Baseline levels of tau predict the subsequent rate of atrophy in Alzheimer disease (AD), researchers have shown in a new imaging study. Renaud La Joie and colleagues recruited 32 individuals who were in the early clinical stages of $A D$ and studied them over a period of 15 months. At baseline, participants underwent PET to assess brain levels and distribution of amyloid- $\beta(A \beta)$ and tau. They also underwent structural MRI, which was repeated at the end of 15 months to assess brain atrophy over the study period. The global intensity of the tau PET signal — but not the $A \beta$ PET signal - at baseline predicted the rate of atrophy over the subsequent 15 months. The distribution of tau pathology at baseline also indicated the spatial pattern of subsequent atrophy. The findings support the idea that tau, rather than $A \beta$, drives neurodegeneration and suggest that tau PET could be a valuable clinical tool for prognosis and clinical decision-making in AD.

ORIGINAL ARTICLE La Joie, R. et al. Prospective longitudinal atrophy in Alzheimer's disease correlates with the intensity and topography of baseline tau-PET. Sci. Transl Med. 12, eaau5732 (2020)

\section{NEURODEGENERATIVE DISEASE}

\section{Detailed genetic link between REM sleep behaviour disorder and synucleinopathies}

Over $80 \%$ of people with REM sleep behaviour disorder (RBD) go on to develop an overt synucleinopathy, such as Parkinson disease (PD) or dementia with Lewy bodies (DLB). However, the precise role of variants in the SNCA gene, which encodes a-synuclein, has not been well studied. In new work, Lynne Krohn and colleagues analysed the association between SNCA and RBD in detail. The researchers sequenced SNCA in 1,076 patients with isolated RBD, 1,013 patients with $P D$, 415 patients with $\mathrm{DLB}$, and 6,155 healthy controls. One variant in the $5^{\prime}$ region of SNCA was associated with RBD and was found to be in linkage disequilibrium with other variants in the same region that are associated with various synucleinopathies. ORIGINAL ARTICLE Krohn, L. et al. Fine-mapping of SNCA in REM sleep behavior disorder and overt synucleinopathies. Ann. Neurol. https://doi.org/10.1002/ana.25687 (2020) 